Mots. Les langages du politique

\title{
La question de la graphie pour la langue kabyle
}

The question of the alphabet for Kabyle language

El tema de la grafía para la lengua cabileña

\section{Chérif Sini}

\section{OpenEdition}

\section{Journals}

Édition électronique

URL : https://journals.openedition.org/mots/22180

DOI : $10.4000 /$ mots. 22180

ISSN : 1960-6001

\section{Éditeur}

ENS Éditions

\section{Édition imprimée}

Date de publication : 9 mai 2016

Pagination : 141-153

ISBN : 978-2-84788-793-8

ISSN : 0243-6450

\section{Référence électronique}

Chérif Sini, «La question de la graphie pour la langue kabyle », Mots. Les langages du politique [En ligne], 110 | 2016, mis en ligne le 09 mai 2018, consulté le 24 avril 2022. URL : http://

journals.openedition.org/mots/22180; DOI : https://doi.org/10.4000/mots.22180 


\section{La question de la graphie pour la langue kabyle}

Le kabyle est la forme de la langue berbère actuellement parlée en Kabylie, au centre nord-est de l'Algérie. De tradition essentiellement orale, il connait, depuis les années soixante-dix, l'accélération de son passage à l'écrit, accompagnant sa redynamisation sociolinguistique en réaction à la politique d'unification linguistique et culturelle du jeune État-nation algérien. Cependant, il se heurte, au niveau institutionnel, à l'épreuve du choix de sa graphie, tifinagh ${ }^{1}$, arabe ou latine. L'examen des arguments pour ou contre chacune des trois possibilités donne à voir le rapport complexe entre la langue et l'écriture en général. Il fait ressortir le poids des conditions socio-historiques et sociopolitiques dans l'émergence de la graphie latine dans l'édition et l'enseignement kabyles, dans l'hésitation à adopter l'alphabet tifinagh et dans le rejet idéologique de la graphie arabe. Il permet ainsi d'analyser les retombées directes du conflit sur la gestion institutionnelle des langues en Algérie et de souligner l'urgence de la démocratisation de cette gestion : compte tenu des mutations sociolinguistiques que connaissent les langues berbères en général, leur survie est menacée à moins de passer par l'écriture et tout ce qu'elle implique comme usage institutionnel et étatique de la langue : école, université, académie, édition, etc.

\section{Le berbère, une langue en voie de disparition?}

En effet, marginalisée depuis la période protohistorique au profit successivement du punique, du grec, du latin, de l'arabe, du français, la langue berbère apparait partout et tout au long de son histoire dans une situation dominée. Exclue de la gestion écrite de la vie des cités même au temps des rois, princes et dynasties berbères (Massinissa, Micipsa, Juba, Almoravides,

1. Vieil alphabet berbère encore en usage chez les Touareg. Les militants kabyles de l'Académie berbère de Paris (1966-1978) l'ont adapté aux spécificités phonétiques du kabyle sans pour autant réussir à en faire le support de produits écrits remarquables. 
Almohades...) et confinée à l'usage domestique et rural, elle n'a pas servi de vecteur d'expression d'une culture savante écrite importante, alors même que l'histoire de la Berbérie retient de grandes figures comme Fronton, Tertullien, saint Augustin, saint Optat, Arnaud, Apulée, saint Cyprien, Lactance, Ibn Tumert, Al Wartilani, Al Warglani, Al Mazudi, etc. Ces auteurs ont écrit dans la langue de ceux qui les ont dominés, parfois pour leur ressembler, souvent pour les combattre, comme c'est le cas notamment des écrivains du $20^{e}$ siècle : JeanEl-Mouhoub Amrouche, Mouloud Feraoun, Mouloud Mammeri, Kateb Yacine, pour n'en citer que quelques-uns. La politique d'arabisation, menée au lendemain des indépendances des pays du Maghreb, vise explicitement l'élimination de leur langue maternelle. De ce fait, le nombre des berbérophones, en lui-même, constitue l'un des enjeux de cette politique conçue et présentée comme le parachèvement d'un processus socioculturel historique. C'est pourquoi les indications relatives aux langues dans les recensements de population de ces pays ne sont pas rendues publiques. Bien qu'approximative, l'évaluation numérique qu'en donne Salem Chaker (1999, p.11; 2004a, p.4045), en s'appuyant sur les sources du $20^{e}$ siècle et en concordance avec les estimations que Jacques Lanfry (1986, p. 45) et Lionel Galand (1989, p. 338) fournissent, atteste du poids démographique « encombrant» des berbérophones. Ces derniers constituent 35 à $40 \%$ de la population marocaine et 20 à $25 \%$ d'Algériens dont les deux tiers sont kabylophones, soit un minimum de 5,92 millions de locuteurs de la langue qui nous intéresse ici, sur une population glo-

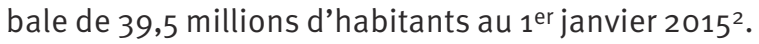

En dehors de l'usage ludique, épistolaire et symbolique de l'écriture tifinagh chez les Touareg (Aghali-Zakara, Drouin, 1997 ; Chaker, 2004a, b), les Berbères n'ont pas connu les effets que l'écriture, cet ensemble de signes visuels servant à noter les sons fonctionnels d'une langue (Février, 1995), a produits ailleurs (Goody, 1986, p. 91) sur les procédés de communication et de pensée et sur les modes d'organisation sociale, politique et économique. La conservation de la mémoire et de la conscience collectives est si peu assurée par l'écriture que les repères culturels et ethniques étaient, jusqu'au siècle passé, situés partout. Même la marginalisation historique du berbère (Camps, 1980), son éparpillement et sa variation géographique sont, en partie, liés à l'absence d'écriture. La pénétration plus ou moins massive de cet « outil de l'intellect » (Goody, 1996) dans les sociétés berbères est concomitante à l'enseignement de l'arabe et du français par les États nouvellement indépendants. Elle a pour corollaire celle des cultures arabe et française. De ce fait, elle valorise les procédés de pensée de ces cultures auprès des écoliers kabyles, touareg, chleuhs, rifains, etc., et prédisposent ces derniers à abandonner leur langue

2. Selon l'Office national des statistiques : chiffres rapportés dans le quotidien d'information El Watan du 29 mars 2015. 
qui, en dehors de sa fonction identitaire, ne leur procure pas la réussite sociale et la promotion professionnelle auxquelles l'arabe et le français leur donnent accès. Au contraire, elle rappelle à ceux qui ont réussi socialement et professionnellement leurs difficultés initiales et les stigmatisations dont ils étaient auparavant l'objet. La tendance à l'abandonner ressort particulièrement en contexte citadin et/ou frontalier de contact entre les langues mais transparait aussi ailleurs, dans ce qui s'apparente à une espèce de rupture dans la chaine de transmission intergénérationnelle de ses éléments culturels : contes, dictons, proverbes, devinettes, berceuses, chants, prénoms... Ce processus est d'autant plus rapide que le berbère est dépourvu d'écriture.

Compte tenu de ces conditions, associées au long processus d'arabisation in vivo d'une grande partie de l'actuel Maghreb depuis le Moyen-Âge, l'extinction de la berbérophonie paraissait, au milieu du $20^{e}$ siècle, irrésistible. En effet, écrit Hildebert Isnard (1966, p.46), « un processus inéluctable fait régresser chaque jour la réalité berbère [...]. L'indépendance nationale acquise, une résistance berbère ne saurait livrer que d'inutiles combats d'arrière-garde contre l'achèvement de l'intégration par l'arabisation ».

\section{Le kabyle : les facteurs d'une singularisation}

Pourtant, au début du $20^{\mathrm{e}}$ siècle, un fait nouveau apparait en Kabylie : des berbérophones revendiquent explicitement leur langue et militent pour en faire un instrument de communication moderne. La revalorisation de leur langue et culture suivra un lent processus de maturation avant de connaitre une formulation politique dans la revendication d'un droit constitutionnel à cette langue. Elle s'accélère brutalement durant les années soixante-dix et devient, depuis, un mouvement populaire porteur d'un projet de société tourné vers la modernité et diamétralement opposé à celui que prône la politique d'arabisation systématique de cette période de l'histoire de l'Algérie indépendante.

La concentration de cette revendication sur la région kabyle tient à la combinaison de plusieurs facteurs parmi lesquels on pourrait citer :

- la prise de conscience, chez les premiers lettrés kabyles en français, de la spécificité de la langue et de la culture berbères (Ouerdane, 1990, p. 21-29);

- l'importance de l'émigration kabyle (en France) dont on connait le rôle dans le mouvement anticolonial et indépendantiste algérien ;

- la densité en population de la région kabyle et la suprématie numérique des kabylophones par rapport aux autres groupes berbérophones d'Algérie réunis;

- la proximité géographique de la Kabylie de la ville d’Alger, capitale politique et administrative de l’État algérien.

Cette concentration se traduit dans les faits suivants :

- la crise antiberbère opposait, en 1948-1949, essentiellement des militants 
nationalistes kabyles à la direction nationale du PPA/MTLD3, dont sera issu le FLN4 (Harbi, 1992);

- la rébellion de 1963-1964 contre la mise en place du régime du parti unique lors de l'indépendance de l'Algérie en 1962, s'est limitée à la région kabyle ;

- la contestation berbère du printemps 1980 était portée par les Kabyles (Ait Larbi, 2011);

- la grève du cartable, durant l'année scolaire et universitaire de 19941995, pour la reconnaissance constitutionnelle de la berbérité n’a touché que cette région et l'enseignement facultatif du berbère, décidé pour mettre fin à ce boycott dans 16 départements où évoluent des berbérophones5, ne sera réellement effectif que dans la région kabyle 6 (Kahlouche, 2000, p. 165) : bien avant l'ouverture démocratique d'octobre 1988, un tissu associatif clandestin y enseignait déjà l'écriture et la lecture au moyen de la graphie latine adaptée au kabyle, objet de description, de standardisation et vecteur d'une production littéraire de plus en plus imposante (Chaker, 2004a, b) ;

- les émeutes meurtrières du printemps 2001 se sont limitées à cette région;

- tous les tests électoraux postérieurs à l'ouverture démocratique d'octobre 1988 distinguent la région kabyle du reste du pays.

Cette singularisation a inspiré, au début de ce $21^{\mathrm{e}}$ siècle, un projet politique d'autonomie régionale. Depuis quelques années, les dirigeants de celui-ci exigent le droit politique à l'autodétermination kabyle7. Cette exigence semble s'accroître à mesure que les autorités du pays tardent à promouvoir le berbère au statut de langue officielle au même titre que l'arabe et à lui donner les moyens juridiques, institutionnels et financiers nécessaires à son aménagement et à sa promotion dans les deux sens, horizontal et vertical. Associée, d'une part, à l'amendement constitutionnel d'avril $2002^{8}$, décidé par le pré-

3. Parti du peuple algérien rebaptisé en 1946 Mouvement pour le triomphe des libertés démocratiques : principale organisation politique nationaliste avant le déclenchement de la révolution algérienne par les indépendantistes du FLN.

4. Front de libération nationale. Issue de l'aile radicale du mouvement nationaliste algérien, cette organisation politique, dotée d'une organisation miliaire (l'Armée de libération nationale), conduira le pays à l'indépendance et une partie de ses membres l'imposera comme parti-État à la manière des républiques socialistes prosoviétiques jusqu'en octobre 1988.

5. Il est difficile de ne pas y voir l'intention de déposséder politiquement les défenseurs kabyles du berbère de l'exclusivité de cette question. En atteste le slogan «Le berbère est le patrimoine de tous les Algériens » des discours présidentiels et ministériels lors de la création du Haut-Commissariat à l'amazighité (la berbérité), qui apparait comme le début de la constitutionnalisation du berbère en Algérie.

6. Selon A. Guedjiba (2014, p. 139), l'enseignement public du berbère dans le Chaouia a gagné du terrain depuis la révision constitutionnelle de 2002 .

7. Ce mouvement politique est sociologiquement difficile à évaluer, pour la même raison que le nombre de berbérophones.

8. Introduction, dans la Loi fondamentale du pays, de l'article zbis portant promulgation du berbère «langue également nationale». 
sident Abdelaziz Bouteflika dans la foulée des émeutes de Kabylie, et d'autre part, aux révoltes populaires en Tunisie, en Égypte, en Lybie, etc., cette exigence est sans doute pour beaucoup dans l'adhésion de l'ensemble de la classe politique visible en Algérie à l'idée de l'officialisation de cette langue, y compris la mouvance islamiste et les appareils politiques au pouvoir qui lui étaient traditionnellement hostiles. En effet, jusqu'au début de ce $21^{\mathrm{e}}$ siècle, seules les organisations politiques dont la base sociologique est essentiellement kabyle, le Front des Forces socialistes (FFS) et le Rassemblement pour la culture et la démocratie (RCD), inscrivaient dans leur programme la promotion du berbère au statut de langue nationale et officielle au même titre que l'arabe'.

Cependant, en conditionnant cette éventuelle promotion, discrètement pour les partis au pouvoir et explicitement pour les partis islamistes, à l'adoption de l'écriture arabe, cette adhésion apparait aux yeux des défenseurs kabyles du berbère comme une instrumentalisation de la question du choix de l'écriture. Ils y voient non pas le souci d'engager un débat en faveur d'une promotion sociale et politique de cette langue, mais la volonté de mener un chantage visant à perpétuer ce différend et à en faire un écueil à toute promotion juridico-politique, alors que pour eux, cette question devrait être réglée par l'usage actuel. Dans le même ordre d'idées, l'apparition relativement massive de l'écriture tifinagh sur les enseignes et autres devantures d'établissements publics, particulièrement après les émeutes de Kabylie au printemps $2001^{10}$, est perçue comme une manœuvre qui, en mobilisant les référents identitaires et affectifs de cette écriture, l'oppose aux écrivains et enseignants kabyles, tous utilisateurs de la graphie latine. Cette suspicion est nourrie par le fait que les autorités publiques, qui tolèrent l'usage de la graphie latine dans l'enseignement scolaire et universitaire publics du berbère, dans l'édition, dans les médias, etc., ne montrent aucune diligence à entériner ce choix. Pourquoi? Que reproche-t-on à l'adoption de cette graphie? Quels arguments formulent ses défenseurs? Pourquoi ces derniers refusent-ils l'adoption de la graphie arabe? Pourquoi n'adoptent-ils pas les tifinagh qui, au moins durant les années soixante-dix, constituaient le symbole de la berbérité, si bien que des citoyens de Kabylie ont été emprisonnés au motif qu'ils en portaient sur eux l'alphabet pour le diffuser discrètement?

9. La présence de cette revendication dans les discours du Parti des travailleurs, dirigé par une arabophone, est en rapport avec l'entourage majoritairement kabyle de la direction nationale de cette organisation.

10. Au début des années quatre-vingt-dix, une version des tifinagh apparait dans la signalétique urbaine à Tizi-Ouzou et à Bejaïa. Après les émeutes de 2001, cet usage connait une relative généralisation aux enseignes de banques, d'écoles, d'universités, d'hôpitaux et d'entreprises publiques. Il ne concerne en revanche que quelques établissements privés, des commerces, généralement. 


\section{La graphie latine : les raisons d'une adoption}

Écrivains, universitaires, enseignants, étudiants et élèves kabyles, utilisateurs actuels de la graphie latine, se considèrent héritiers de ce choix (Sini, 2007). En y adhérant, ils assument sans ambigüité les implications didactiques, politiques et idéologiques qui le motivent. Il s'agirait, d'une part, d'éviter les confusions qui découleraient de l'écriture du kabyle avec la graphie arabe en raison d'un univers scolaire arabisé, et, d'autre part, de marquer leur adhésion à la sphère de civilisation euro-occidentale, dont l'expansion planétaire s'accompagne de celle des outils de la grammatisation contemporaine que sont l'espagnol, le français ou l'anglais (Auroux, 1994) : il y a là l'idée d'une universalité de cette graphie qui serait transférable au kabyle, comme si un texte kabyle écrit dans cette graphie gagnait la possibilité d'être lu et compris par un francophone, un anglophone, un hispanisant, du fait de l'identité des graphies.

On voit cependant mal en quoi l'universalité supposée de la graphie latine faciliterait l'apprentissage du kabyle, dont il est à peine besoin de rappeler le statut de langue en danger de disparition, qui n'intéresse que ses défenseurs. En outre, la similitude des composants de l'alphabet latin n'implique pas celle des sons fonctionnels qu'ils sont chargés de rendre selon les règles orthographiques spécifiques des langues. Par ailleurs, l'argument de l'universalité de la graphie latine entre en contradiction avec la fonction identitaire qui, seule, permet encore au kabyle de survivre, et semble à cet égard peu cohérent avec le rejet de la graphie arabe au motif justement que son adoption ne garantirait pas une distanciation identitaire suffisante du kabyle vis-à-vis de l'arabe. Il faut ajouter à ces remarques que l'aspect supposé pratique et facile de l'utilisation scripturaire et surtout électronique de cette graphie ne relève pas d'une qualité intrinsèque à cette graphie mais tient à l'effort consenti par les utilisateurs.

L'histoire des formes et des normes des langues montre néanmoins que l'usage s'accommode peu des reconstructions et abstractions des observateurs avertis, et qu'il a des effets, rarement recherchés, sur les pratiques langagières en général. Cet aspect historique, c’est-à-dire concret et vérifiable, de la grammatisation en domaine kabyle constitue en revanche un argument difficilement discutable : l'accumulation de productions écrites en kabyle au moyen de la graphie latine, et notamment les manuels et supports pour l'enseignement du kabyle, les exemples et suggestions pour la création littéraire et artistique directement composés en kabyle, traduits et adaptés au kabyle pour servir la constitution du savoir sur le kabyle en kabyle, rendent difficile sa remise en cause, et ce d'autant plus que la production écrite en kabyle dans les graphies tifinagh et arabe est insignifiante.

En fin de compte, l'argument de la scientificité de la graphie latine, qui est utilisée dans les départements universitaires de Tizi-Ouzou, de Bejaïa et de 
Bouira, par l'Inalco (Paris) mais aussi dans les travaux de Mouloud Mammeri (Sini, 2011, p. 611), auxquels font écho des linguistes berbérisants de renom à l'instar de Salem Chaker, Ramdane Achab, Kamel Nait Zerrad, Amina Mettouchi, etc., explicite plus qu'il ne justifie l'adoption de cette graphie, sinon en permettant de regarder de plus près les possibilités d'adaptation des deux autres graphies tifinagh et arabe. Celles-ci, du moins théoriquement, sont aussi envisageables, pour peu qu'on dissocie la graphie arabe de la sacralité qui rend sa réforme problématique (Meynet, 1971) et qu'on accepte de profondes modifications formelles compromettant l'authenticité originelle de la graphie tifinagh, en substituant aux nombreux caractères, constitués de cercles, de points et de barres susceptibles de produire des confusions, d'autres plus propices à la fonction distinctive et communicative de l'écriture alphabétique. Dans le cas de l'arabe, l'exigence est à la fois idéologique et politique, ce qui empêche sa réforme et, par ricochet, en fait une graphie techniquement inadéquate aux particularités linguistiques kabyles. Pour les tifinagh, l'exigence est plus technique mais elle revêt des implications symboliques qui en découragent l'adoption, et ce en dépit de la voie officielle dans laquelle le berbère est engagé au Maroc, particulièrement depuis qu'il a été promu au statut de langue officielle, en vertu de la révision constitutionnelle de 2011.

Pour quelle raison alors l'adoption de la graphie latine par les utilisateurs du kabyle n'est-elle pas entérinée au niveau institutionnel? On lui reprochait son caractère français, contraire aux objectifs de la politique d'arabisation. Mais avec la redynamisation politique que connait le français en Algérie depuis l'arrivée d'Abdelaziz Bouteflika à la magistrature suprême de l'État, cet argument apparait fragile et sans effet. Faire percevoir le français derrière cette écriture constituait, en réalité, la justification d'une attitude de principe hostile à la vitalité du berbère qui, en tant qu'élément anthropologique et culturel, perturbait les fondements d'un projet de mystification revendiquant une ethnicité exclusive : l'arabité. Le réglage idéologique du sens de l'ethnonyme de ce nom dans les productions discursives en accord avec la ligne idéologique au pouvoir rimait, en effet, avec une conception passéiste et rétrograde de l'arabité. Naturellement, l'arabe, en tant que langue et civilisation, n'a rien à voir avec ce positionnement et les implications qui en étaient déduites. Il est victime du fait que c'est en son nom qu'on a entrepris ce qu'il convient d'appeler un acte politique intentionnellement « linguicide», qui a au moins renforcé pour ne pas dire suscité le rejet de la graphie arabe par les kabylophones.

Il faut néanmoins revenir sur cet argument selon lequel l'adoption de la graphie latine pourrait servir d'instrument d'occidentalisation du kabyle et du berbère en général.

Comme les langues, les écritures sont en effet porteuses de cadres de perception liés à l'histoire sociale et matérielle de leur constitution et à l'usage qu'on en fait selon les données technologiques des époques et les aspirations 
des utilisateurs. En réalité, ce sont ces derniers qui décident ce qu'en faire, comme le montre la révolution kamélienne à la fin de l'empire Ottoman. En substituant à l'écriture arabe une graphie d'inspiration latine, Atatürk a procédé en même temps à la laïcisation de la gestion politique de la société ottomane en fondant la République turque. La réforme de l'écriture a participé à ce processus de modernisation du turc qui consistait, par exemple, à emprunter des éléments néologiques de langues européennes plutôt que de l'arabe ou du persan. Le transfert à la langue turque de la modernité que représentait à cette époque le français, impliquait alors en effet l'abandon des éléments de cette langue issus de l'arabe et jugés incompatibles avec la visée moderniste dans laquelle était engagée la gestion politique de la société turque dans sa globalité.

Les implications sur le kabyle de l'adoption de la graphie latine sont de cet ordre avec, en plus, la proximité interculturelle qu'offre actuellement la mondialisation des moyens aussi bien de la communication que de la constitution des savoirs, ainsi que des procédés de pensée et de gestion de la connaissance. À ce sujet, on ne peut pas ne pas constater déjà l'impact qu'a produit, sur les utilisateurs kabyles de cette graphie, l'idée d'une occidentalisation du berbère, dans laquelle les adversaires de la berbérité voyaient, en réalité, les germes de la contestation politique. Cela se vérifie, d'une part, dans la démarcation du type d'écriture adopté, et qui est de tendance phonologique plutôt qu'orthographique et étymologique comme en français, ou phonétique comme en espagnol, etc. D'autre part, cette démarcation est visible dans le choix de la néologie lexicale et terminologique (Sini, 2009; Achab, 2014) plutôt que de l'emprunt pour rendre en kabyle les concepts et autres idées ou réalités que la forme du kabyle traditionnel n'exprime pas en raison de la marginalisation historique que nous avons rappelée plus haut. Cependant, il est difficile d'admettre, avec le recul, que les adversaires de l'adoption de la graphie latine avaient réellement le souci de préserver le berbère de cette francisation/occidentalisation. C'est le regard kabyle tourné vers le mode de vie occidental et donc en rupture avec le leur, aussi bien dans le rapport à la religion, le statut social de la femme, etc., déjà ancré dans les traditions orales locales, qu'ils cherchaient à réorienter selon les modalités répressives de la pensée unique, que la politique d'arabisation, associée au socialisme à la soviétique, était chargée de concrétiser.

\section{Les tifinagh ou les motifs d'une hésitation}

Perçu comme un des repères d'histoire et d'identification berbères, l'alphabet tifinagh est actuellement très peu utilisé dans l'édition et l'enseignement kabyles, même si ce sont les militants kabyles de l'Académie berbère de Paris (1967-1978) qui l'ont remis à l'honneur alors qu'il était sorti de l'usage depuis le Haut Moyen Âge! Symbole de la berbérité dans le milieu kabyle des années 
soixante/quatre-vingt et, à ce titre, strictement interdit par les autorités politiques algériennes de l'époque, cet alphabet était utilisé dans l'édition et l'enseignement à titre exceptionnel mais il avait des effets subversifs sur l'idéologie de l'ordre linguistique mis en place. Discrètement concurrencé par l'alphabet latin, durant les décennies soixante-dix/quatre-vingt, puis, entièrement supplanté par ce même alphabet à partir des années quatre-vingt-dix, aussi bien dans l'édition que dans l'enseignement associatif, il conservera néanmoins son attrait affectif parmi les jeunes générations kabyles pour qui la berbérité suppose les tifinagh, comme si cette graphie faisait partie intégrante du berbère alors que nous sommes là en présence de deux moyens de communications à la fois distincts et interdépendants, comme l'atteste l'histoire de l'écriture (Février, 1995). Cette équation est si évidente pour cette jeunesse que, lors de l'introduction expérimentale du berbère dans le système éducatif algérien durant l'année scolaire 1995-1996, beaucoup d'enseignants de berbère en Kabylie ${ }^{11}$ rapportaient l'étonnement et la déception des élèves de ne pas voir cette graphie au tableau et l'hésitation de la majorité d'entre eux à adhérer à l'option latine de leurs enseignants (Sini, 1997, 2007). Admiratifs devant cette attitude vis-àvis des tifinagh, ces enseignants avouaient des difficultés liées à cette question du choix graphique durant ces premières années de l'enseignement public du kabyle, à chaque début d'année, avec de nouveaux élèves pour qui « le berbère c'est tifinagh » (Sini, 2007, p. 345). Si bien que ces enseignants se devaient de formuler avec beaucoup de prudence les raisons pour lesquelles ils n'utilisaient pas «l'alphabet des ancêtres» (Sini, 2007, p. 332) et se seraient entendus pour dispenser aussi des cours d'alphabétisation en tifinagh, avec un non-dit, celui des limites de cette écriture. Celles-ci tiennent à l'aspect multiforme de cette graphie ${ }^{12}$, à ses imprécisions techniques, aux risques de confusion entre ses innombrables caractères constitués de points, de barres, de cercles avec ou sans point à l'intérieur, au manque cruel de produits culturels écrits avec cette graphie, etc.

Tout en regrettant de ne pas avoir reçu de formation dans cette graphie, ces enseignants de français, d'anglais, d'arabe, etc., reconvertis ad hoc en enseignants de berbère, se retrouvent ainsi au centre d'un débat biaisé. Cette introduction du berbère à l'école semblait en effet constituer une subtilité politique en devenir : l'assimilation intentionnelle de la graphie latine au français, voire à la francophilie, devait décourager l'assiduité au cours de berbère, d'autant plus que celui-ci était facultatif et programmé en fin de journée, et surtout en année d'examen national... Le lien entre la graphie tifinagh et le berbère était donc incompatible avec cette manœuvre qui permettait à ses auteurs, fondamentalement hostiles à la berbérité, de reprendre ce qu’ils avaient été amenés

11. Abdenaceur Guedjiba (2014) rapporte les mêmes réactions dans le Chaouia, au sud du Constantinois.

12. Plusieurs alphabets : les tifinagh traditionnels, les néo-tifinagh et le lybico-tifinagh de S. Chaker (1994). 
à céder pour gagner l'adhésion de la région kabyle au combat contre la violence de la décennie 1990-2000, qui menaçait d'effondrement total les structures de l'État. Le débat était en outre discrètement orienté non pas entre les deux alphabets, latin et tifinagh, en concurrence dans cet espace de mobilisation sociopolitique et éditorial pour le berbère, mais entre l'alphabet latin et l'arabe. Selon cette manœuvre, le premier devait représenter la langue et la culture de l'ancien colonisateur et ennemi de la religion musulmane, le second la langue de la souveraineté nationale et surtout du Coran...

L'évolution des rapports de forces politiques en faveur du berbère à la suite de cet épisode de l'histoire de la promotion du berbère en Algérie et au Maroc, a cependant fait échec à cette volonté de limiter le débat entre les graphies latine et arabe, faisant basculer les partisans de la graphie arabe dans le camp de ceux des tifinagh, d'autant plus que ce choix était déjà en 2003 celui des autorités du Royaume chérifien, d'accord avec les autorités algériennes sur le principe d'ostracisme à l'égard du berbère. C'est ce qui explique aussi l'apparition, plus ou moins massive, de cette graphie dans les enseignes et devantures d'établissements étatiques en Kabylie au lendemain de la promotion du berbère au statut de «langue également nationale » en avril 2002, qui a coïncidé avec les prémisses de l'adoption de cet alphabet par l'Institut royal de la culture amazighe au Maroc (Ircam). Curieusement, cette apparition se limite à la région kabyle et ne concerne pas les régions touarègues, berceau originel des tifinagh, où cette graphie pourrait pourtant servir le marquage de ce territoire... Cette mesure semble ainsi rappeler que la promotion juridique du berbère doit s'accompagner de l'adoption de la graphie tifinagh. Le doute plane donc sur les véritables motivations de ce choix à la fois discret et surprenant quand on se rappelle l'attitude des pouvoirs publics des années soixante-dix vis-à-vis de cette écriture. Ce qui fait dire à des écrivains kabyles en kabyle (Sini, 2007) que l'exploitation des éléments identitaires liés aux tifinagh vise à discréditer auxyeux des populations les auteurs en berbère qui, eux, utilisent les caractères latins. On comprend dès lors pourquoi la graphie tifinagh paraisse désormais suspecte à ces derniers après avoir été le repère visible de la berbérité, suspicion qui rend la légitimation de cette graphie plus difficile : elle est perçue comme étant orientée contre la graphie latine, c'est-à-dire contre les auteurs kabyles, tous utilisateurs de cette graphie, et dont le capital de confiance et de notoriété est à la hauteur des aspirations kabyles à demeurer kabyles et à expliciter la kabylité dans l'ensemble juridique national pour en faire un projet moderne, sur le modèle démocratique en vigueur dans l'espace euro-occidental actuel.

\section{La graphie arabe ou les causes du rejet}

La flexibilité des formes des lettres arabes, en position initiale, médiane ou finale, exige un apprentissage supplémentaire et rappelle quelque peu l'ef- 
fort à fournir pour maitriser la cursive en écriture d'inspiration latine. Des créations de caractères et/ou des modifications internes sont ainsi nécessaires pour adapter l'arabe au kabyle. Pour les textes manuscrits, cela implique une surcharge de la ligne d'écriture et une certaine lenteur dans l'exécution, particulièrement quand il s'agit d'indiquer la vocalisation. La saisie informatique nécessite quant à elle un clavier spécial pour ne pas insérer des équivalents spécifiques à partir des caractères spéciaux qu'offre l'ordinateur, comme cela se fait encore avec la graphie latine, ce qui demande une certaine gymnastique et du temps alors même que la révolution numérique en cours exige de la rapidité. L'exemple des sons tendus, comme en langue arabe, est parlant : on les transcrit au moyen de la shadda arabe, le chiffre 3 couché sur le dos auquel il convient de rajouter en suscrit et en souscrit le signe de vocalisation pour rendre respectivement /a/, /u/ et/i/.

Du point de vue matériel donc, la graphie arabe, à l'instar de l'écriture tifinagh et de l'alphabet latin, est aussi adaptable au kabyle pour peu qu'il y ait suffisamment de volonté pour entreprendre cet exercice plus ou moins résolu avec la graphie latine. Cela exige évidemment un engagement collectif et individuel pour combler à la fois le déficit intellectuel en la matière, mais aussi et surtout pour réparer le préjudice identitaire, culturel et linguistique commis à l'encontre de la berbérité au nom de la généralisation de l'emploi de la langue arabe. C'est donc également un exercice étatique que les décideurs politiques doivent réussir dans le cadre de la réconciliation avec la Patrie dans son authenticité et sa diversité et dans la perspective de leur abstraction en une nation politique unie.

En définitive, les arguments formulés pour ou contre l'adoption de la graphie arabe sont aussi discutables que ceux qui concernent les alphabets latin et tifinagh. C'est le cas, par exemple, des ressemblances structurales entre le kabyle et l'arabe du fait de leur appartenance au groupe de langues chamitosémitiques. Les défenseurs du kabyle cherchent justement à rétablir les distances entre les deux langues pour atténuer la menace qui pèse sur le kabyle de se voir assimilé à l'arabe du fait de l'hégémonie sociopolitique de ce dernier. Les éléments culturels de la graphie arabe pourraient, en effet, avoir des incidences sur les modes d'équipement du kabyle, comme on peut déjà le constater en Turquie ottomane, au Pakistan ou en Afghanistan. Ces effets sont à peu près l'équivalent de ceux des premières étapes de la grammatisation, avant que la langue écrite au moyen des caractères latins n'acquiert son autonomie visà-vis des langues qui servent sa description, son outillage et son équipement intellectuels. Cette promiscuité culturelle est d'autant plus incompatible avec l'orientation idéologique des défenseurs kabyles du berbère qu'elle est actuellement associée à la restriction des droits des citoyens en matière de culte et d'interprétation du monde physique et spirituel. Ces libertés individuelles et collectives auxquelles aspirent ces locuteurs s'accommoderaient-elles de la 
sacralisation de la graphie arabe, alors même que la réforme de la langue arabe est difficilement envisageable dans le contexte politique mondial actuel?

Dans la mesure où ce n'est pas de la langue arabe dont il s'agit mais bien de la graphie arabe, son adaptation au kabyle serait envisageable si les partisans de cette graphie étaient animés des mêmes intentions politiques que les défenseurs du kabyle, utilisateurs du latin. Mais leur proximité avec la politique linguistique et la politique tout court du pays la rend d'autant plus difficile que la grammatisation du kabyle s'est développée, jusqu'à la fin du siècle dernier, en dehors des circuits et des institutions de l'État et surtout en opposition frontale avec l'arabisation. Le défi à relever pour rétablir cette confiance rompue au nom de l'unification et de l'homogénéisation culturelle est donc immense.

À l'heure actuelle, les différentes améliorations que l'usage a apportées à l'adoption de la graphie latine, depuis plus d'un siècle d'activité de grammatisation, font de celle-ci l'écriture actuelle du kabyle. Sans ancrage réel dans l'enseignement ni dans l'édition en kabyle, l'alphabet tifinagh est loin de concurrencer cette option. Mais derrière le conflit sur la graphie à adopter pour écrire le kabyle se profile, en fait, celui que soulève la gestion des langues en Algérie. La politique d'unification culturelle et linguistique a généré un malaise identitaire que les berbérophones de Kabylie expriment, ici, par le rejet de la graphie arabe. Celle-ci symbolise pour eux une politique qui, en raison de son fondement idéologique, ne pouvait pas tolérer la diversité linguistique et encore moins encourager la production en kabyle, y compris dans la graphie arabe. L'ouverture récente de cette politique aux autres langues réussira-t-elle à convaincre les défenseurs kabyles du berbère de la réalité de la démocratisation de la gestion de ces langues?

\section{Références}

ACHAB Ramdane, 1998, Langue berbère. Introduction à la notation usuelle en caractères latins, Paris, Hoggar.

- 2014, L'aménagement du lexique berbère de 1945 à nos jours, Tizi-Ouzou, Achab. Ait-LARBI Arezki, 2011, Avril 8o, Alger, Koukou.

Aghali-Zakara Mohamed, Drouin Jeanine, 1997, «Écriture libyco-berbère. Vingt-cinq siècles d'histoire", L'aventure de l'écriture. Naissances, A. Zali, A. Berthier éd., Paris, Bibliothèque nationale de France, p. 98-111.

Auroux Sylvain, 1994, La révolution technologique de la grammatisation, Liège, Mardaga. CAMPS Gabriel, 1980, Berbères aux marges de l'Histoire, Toulouse, Hespérides.

CHAKER Salem, 1994, "Pour une notation à base tifinagh », Études et documents berbères, nº 11, Aix-en Provence, Édisud, p. 31-42.

- 1999, "La langue berbère. Entre marginalisation et affirmation», Langues $d u$ Maghreb et du Sud méditerranéen, Cahiers de sociolinguistique, nº 4, p.11-24. 
— 2004a, «Kabylie : La langue», Encyclopédie berbère, vol. 26, Aix-en-Provence, Édisud, p. 4045-4066.

- 2004b, "Langue et littératures berbères», https://www.clio.fr/BIBLIOTHEQUE/ langue_et_litterature_berberes.asp (consulté le 8 décembre 2014).

FÉVRIER James, 1995 (1948, 1958 et 1984), Histoire de l'écriture, Paris, Payot.

GALAND Lionel, 1989, "Les langues berbères », Language Reform. History and Futur / Réforme de langues. Histoire et avenir, C. Hagège, I. Fodor éd., vol. 6, Hambourg, H. Buske, p. 335-353.

Goody Jack, 1986, La logique de l'écriture. Aux origines de la pensée humaine, Paris, Armand Colin.

- 1996, L'homme, l'écriture et la mort. Entretien avec Pierre-Emmanuel Dauzat, Paris, Les Belles Lettres.

GuedjIBA Abdenaceur, 2014, "Enseignement du tamazight dans le massif de l'Aurès. Présentation/analyse d’une expérience», Dialogue des cultures, n60, Lannou, FIPF, p.133-147.

HARBI Mohamed, 1992, L'Algérie et son destin. Croyants ou citoyens?, Paris, Arcantères.

ISNARD Hildebert, 1966, Le Maghreb, Paris, PUF.

MEYNET Roland, 1971, L'écriture arabe en question. Les projets de l'Académie de langue arabe du Caire de 1938 à 1968, Beyrouth, Dar El Machreq.

KAHLOUCHE Rabah, 1999, «De la vitalité du berbère en Kabylie. Aperçu socio-historique », Langues du Maghreb et du Sud méditerranéen. Cahiers de sociolinguistique, n ${ }^{\circ} 4$, p. 37-45.

- 2000, "L'enseignement d'une langue non aménagée, au statut indéfini : le berbère en Algérie », Les langues en danger, Mémoires de la société de linguistique de Paris, nouvelle série, t. VII, p. 157-168.

LANFRY Jacques, 1986, » Les Berbères, leur langue, leur culture. Un renouveau contemporain », Études et documents berbères, $\mathrm{n}^{\circ}$ 1, p. 41-60.

MAMmeri Mouloud, 1976, Tajerrumt $n$ tmaziyt (tantala taqbaylit), Paris, Maspero.

Ouerdane Amar, 1990, La question berbère dans le mouvement national algérien, 1926-1980, Québec, Sillery / Paris, Septentrion.

SıNı Chérif, 1997, Analyse des attitudes de locuteurs amazighophones à l'égard des trois graphies en usage, mémoire de magister sous la dir. de R. Kahlouche et de J.-B. Marcellesi, Université d'Alger.

- 2007, Contribution à l'analyse des raisons du choix d'une graphie pour écrire le kabyle, thèse de doctorat en sciences du langage sous la dir. de F. Laroussi, Université de Rouen.

- 2009, «Des romanciers en kabyle face à la néologie lexicale », Berberstudies, n² 25 , p. 205-214.

- 2011, "La notion de scientificité dans le choix d'écrire le kabyle au moyen des caractères latins », Parcours berbères, Mélanges offerts à Paulette Galand-Pernet et Lionel Galand pour leur 90e anniversaire, A. Mettouchi éd., Berber studies, n 33 , p. 611-622. 